- Periapical ameloblastoma presents as a periapical radiolucency below the roots of a second right mandibular molar

- The periapical ameloblastoma recurred after inadequate treatment

- The importance of prompt, accurate diagnosis and management is discussed

- Differential diagnosis prior to treatment is mandatory - a tissue biopsy should be undertaken

\title{
Periapical ameloblastoma - A case report
}

\author{
M. H. K. Motamedi ${ }^{1}$
}

\begin{abstract}
Ameloblastomas, although often benign, are locally aggressive odontogenic lesions. Presentation of this lesion as a pericoronal radiolucency associated with an impacted tooth is well documented. ${ }^{1-4}$ Presentation of this lesion as a solitary periapical radiolucency associated with a non-impacted tooth however, is rare. ${ }^{1-4}$ Periapical presentation of this lesion may confuse practitioners. ${ }^{3}$
\end{abstract}

Small incipient lesions may be mistaken for a common periapical granuloma or cyst and the tooth may be treated endodontically or extracted with the lesion going undiagnosed or inadequately treated. Differential diagnosis prior to definitive treatment is mandatory and necessitates a tissue biopsy as this lesion requires to be treated more aggressively than other benign periapical lesions. This report presents an ameloblastoma occurring as a periapical lesion below the roots of the second right mandibular molar which recurred after inadequate treatment, and discusses the importance of prompt, accurate diagnosis, and definitive management.

\section{CASE REPORT}

A 20-year-old male presented on 7 September 1996 with a small swelling in the vestibular area of the right second mandibular molar region. Clinically, the area was slightly tender and the tooth had grade two mobility. There was no nerve deficit or adenopathy in the head and neck. Vitality testing of the tooth revealed the tooth to be vital. Radiographically, the tooth had a relativelydefined radiolucent lesion in the periapex

\footnotetext{
${ }^{1}$ Associate Professor of Oral and Maxillofacial Surgery, Clinic of Oral and Maxillofacial Surgery and Trauma Research Center, Baqiyatallah University of Medical Sciences, and Attending Surgeon, Azad University of Medical Sciences, Tehran, IR Iran

Correspondence to: Africa Expressway, Golestan St., Giti

Blvd. No. 11 Tehran, 19667, Iran

E-mail:Motamedical@lycos.com
}

Refereed Paper

Received 27.02.01; Accepted 10.04.02

๑ British Dental Journal 2002; 193: 443-445 with resorption of the mesial and distal root apices (Fig. 1). The medical history was not significant and the patient was in good general health.

The tooth was extracted by his dentist and the lesion was removed and submitted for pathologic examination. Several millimetres of the bony socket was also ground away using a round bur before wound closure perhaps because a fibroosseous lesion, or a keratocyst was suspected. The pathology report however, diagnosed a plexiform ameloblastoma. The patient failed to return for additional treatment because he thought that the treatment rendered was adequate.

Signs of recurrence, became evident 2 years later when swelling recurred, and the patient was then referred. Upon admission, clinical examination of the patient was notable for facial asymmetry and a firm swelling in the area of the right buccal vestibule adjacent to the previous extraction site in the area of the right second mandibular molar.

It was apparent from examination of the patient's initial radiographs, that the buccal cortex was expanded and eroded and the mandibular bone was invaded down to the dental canal (Fig. 2). CT scans were done to better define the extent of the lesion (Fig. 3). An intraoral biopsy was repeated under local anesthesia which confirmed the diagnosis of a plexiform ameloblastoma (Fig. 4) and the patient was scheduled for en bloc surgical resection of the involved mandible.

Under general anesthesia, via a routine intraoral and extraoral (Risdon) supraperiosteal approach to the mandible an AO $2.7 \mathrm{~mm}$ reconstruction plate was first adapted to the mandibular border. The plate was then unscrewed and the tumor resected en bloc along with the overlying soft tissues and the alveolar
Fig. 1 Panoramic radiograph of the patient taken 7 September 1996 demonstrating a radiolucent lesion below the roots of the second right mandibular molar with extensive root resorption, $A$. Coronal CT scan depicting the periapical lesion, $\mathrm{B}$

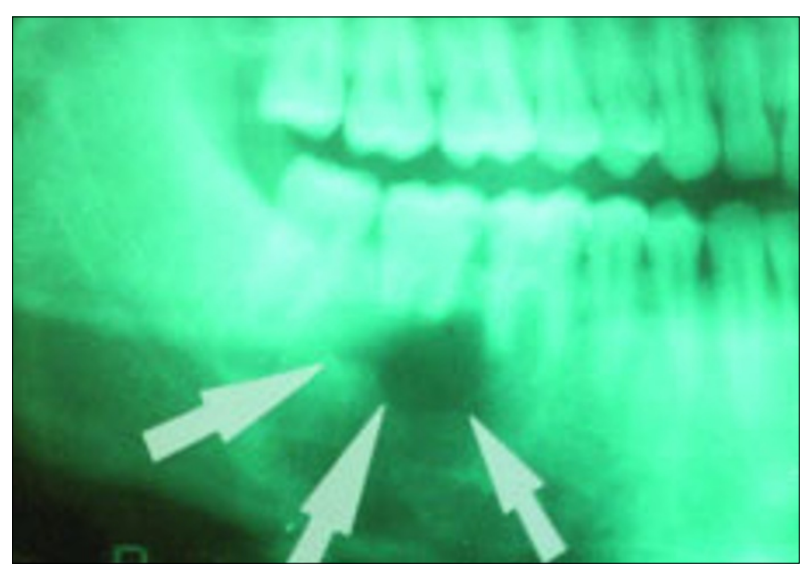




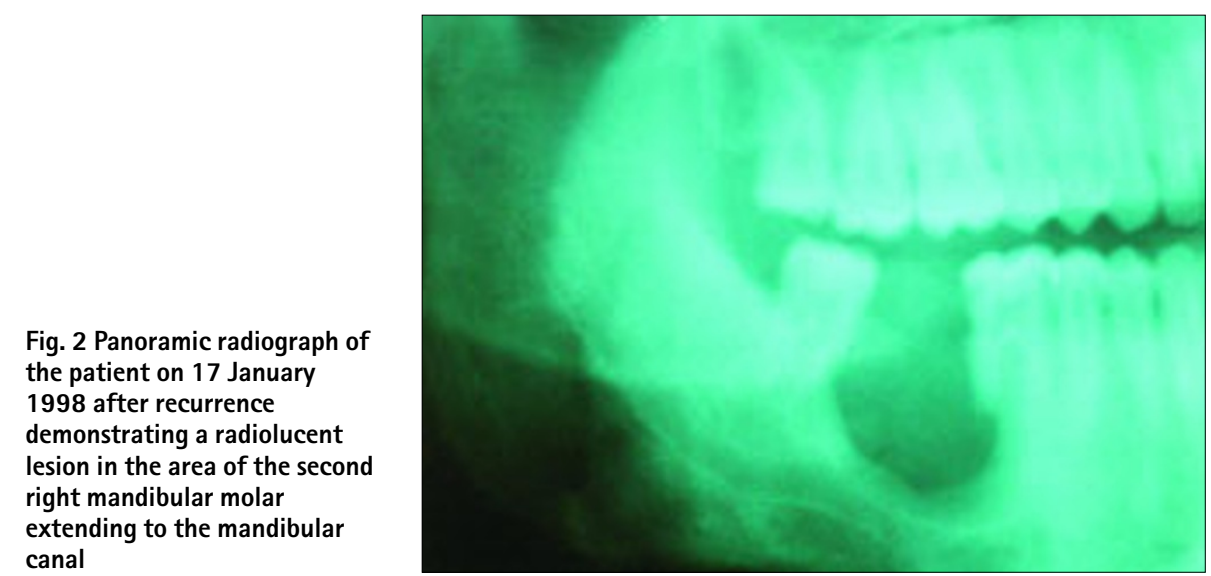

canal
Malassez) in the periodontal ligament, or remnants of the dental lamina (rests of Serres). Ameloblastomas may be confused clinically with other jaw lesions, and occasionally with infiltrating neoplasms of the maxillary sinus, particularly those of salivary gland origin. ${ }^{8}$

\section{Clinical presentation}

Ameloblastomas typically occur around the age of 40 years; children are rarely affected. Odontogenic tumours that are more likely to appear in children are adenomatoid odontogenic tumour and ameloblastic fibroma (both of which have decidedly different microscopic features and biologic behaviors). Ameloblastoma may appear anywhere in the jaws, although the molar-ramus area of the mandible is the favored location. ${ }^{8}$ Affected patients are asymptomatic, and lesions are usually discovered during routine radiographic examination or because of jaw swelling.

\section{Histopathology}

Microscopically, ameloblastomas mimic the ameloblasts and stellate reticulum that ordinarily give rise to enamel. Numerous histologic patterns may be seen in ameloblastomas. The common denominator to all ameloblastomas is well-differentiated palisaded cells found around the periphery of nests, strands, and networks of epithelium. Nuclei of the palisaded cells are typically polarized away from the basement membrane. Budding of epithelium from these proliferative nests and strands is also characteristic of this lesion. Palisading cells and budding epithelium are found in all microscopic subtypes (follicular, cystic, plexiform, desmoplastic, acanthomatous, granular cell). ${ }^{8}$

\section{Radiographic Features}

Radiographically, ameloblastomas usually appear as unilocular or multilocular pericoronal radiolucencies often associated with impacted teeth. They characteristically mandibular and mental nerve stumps. The wound was closed in layers and a suction drain was placed.

The patient's subsequent course was unremarkable. Tumour-free margins were confirmed by the pathologist. The patient underwent a mandibular reconstruction 2 months later and an iliac cortico-cancellous bone graft block from the medial aspect of the right anterior iliac was harvested along with generous bone marrow. It was placed firmly into the defect between the remaining mandibular stumps and secured to the plate with two bicortical $2.7 \mathrm{~mm}$ screws. Graft take was successful and the patient's post-operative course was uneventful. The patient is doing well 6 years post-operatively.

Fig. 4 Photomicrograph of the specimen. Plexiform ameloblastoma (H CEE x 200) Note the large anastomosing sheets of epithelium bounded by columnar ameloblastlike cells, and surrounding the stellate reticulum

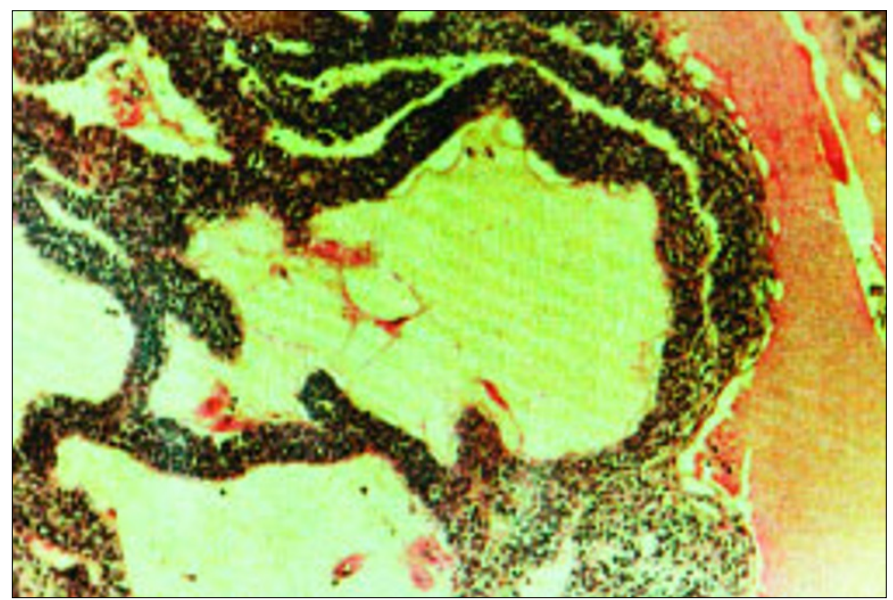


exhibit slow but unrelenting and destructive growth. Periapical presentation of an ameloblastoma is very rare. ${ }^{1-3}$ Thus, it is usually not suspected when it occurs in this location. Teeth presenting with periapical ameloblastomas are often vital, however, there may be associated pulpal pathosis in which case diagnosis may be more difficult.

\section{Management}

Proper diagnosis and management of ameloblastomas cannot be over-emphasized because of the high recurrence rate demonstrated by this lesion. ${ }^{4-8}$ Inadequate diagnosis and treatment of this lesion (ie as a periapical cyst or granuloma) although followed by initial healing, may lead to a recurrence years later, but with more aggressive behavior.

Ominous signs such as root resorption and tooth mobility should not go unnoticed. Paresthesia which may be prevalent in larger lesion should also raise suspicion as to an aggressive or malignant lesion.

It is imperative therefore, that, after clinical and radiographic evaluation of a patient with a periapical lesion, diagnosis be confirmed by obtaining a specimen and sending it for microscopic examination (even if the lesion is small and or associated with a pulpal-involved tooth) so that prompt appropriate treatment may be rendered.

An incisional or excisional biopsy may be done depending on the size of the lesion and its clinical features. ${ }^{1,4}$ An incisional biopsy is advantageous if a representative specimen can be obtained. ${ }^{1}$ This will provide the clinician with a definitive diagnosis and allow for an appropriate workup before developing a therapeutic protocol.
An exception is a patient with a small, unilocular lesion in which the clinical impression is an odontogenic cyst or firbro-osseous lesion where an excisional biopsy is usually performed. ${ }^{1,4}$

If subsequent microscopic examination confirms an ameloblastoma, the clinician must then decide on additional surgery and take the necessary measures.

Ameloblastomas require at least surgical excision, if not resection. Recurrence rates of 50\% to $90 \%$ have been associated with lesions treated by curettage alone and should by no means be underestimated. The growth characteristics of ameloblastomas vary from other benign lesions and tumor resection is often advocated regardless of the type of ameloblastoma. ${ }^{1-8}$ This tumor is infiltrative, and locally invasive, and growth occurs in pathways of least resistance infiltrating through cancellous bone. $^{6}$

Enlargement causes expansion and erosion of cortical bone as well as lamellar thinning. Because of this microscopic infiltration of bone, treatment also necessitates removal of some of the normal bone adjacent to the periphery of the tumour. The appropriate amount of normal bone beyond a radiographic boundary required for a tumour-free margin has not been definitively established.

Marx et al. advocate resection of at least $1 \mathrm{~cm}$ of normal-appearing bone beyond the radiographic tumor margin. ${ }^{6}$ Other clinicians, such as MacIntosh, recommend $2 \mathrm{~cm}$ or more. When the tumour has perforated bone, removal of adjacent soft tissue extending to the next adjacent anatomic boundary must be performed to ensure complete tumour-free soft tissue margins. ${ }^{1,4}$
Preservation of the inferior alveolar nerve is not prudent when it is involved by the tumour, and it should be resected en bloc with the specimen in such cases. This may have been the cause of the recurrence in our case. Immediate nerve reconstruction after extirpation of the tumour restores lower lip sensation for the patient. It is most important to emphasize, to both the clinician and the patient, the need for a definitive treatment protocol and lifetime periodic follow-up for detection of recurrence as even a five year tumour-free period does not necessarily mean a cure. ${ }^{7-8}$

The author would like to thank Dr. Kowsari and Ms. Haghighi from the Department of Pathology for their kind help with the photomicrographs, and also the Radiology Department staff for their help with the radiographs.

1. Wood N K, Goaz P W, Jaocobs M C. Periapical Radiolucencies. In Wood N K, Goaz P W. Differential Diagnosis of Oral and Maxillofacial Lesions. 1997, Philadelphia: CV Mosby, pp252-277.

2. Williams TP. Aggressive odontogenic cysts and tumors. Oral Maxillofac Surg Clin NAm 1997; 9: 332-335.

3. Hollows P. Fasanmode A, Hayter J P. Ameloblastoma. A diagnostic problem. Br Dent J 2000; 188: 243-244.

4. Williams T P. Management of Ameloblastomas. J Oral Maxillofac Surg 1993: 51: 1064-1070.

5. Motamedi M H K, Khodayari A. Cystic ameloblastomas of the mandible. Med I Islamic Rep Iran 1992; 6: 75-79.

6. Marx R E, Smith B H, Smith B R. Swelling of the retromolar region and cheek associated with limited mouth opening. J Oral Maxillofac Surg 1993; 51: 304

7. Macintosh R B. Aggressive surgical management of ameloblastoma. Clin Oral Maxillofac Surg 1991; 3: 73

8. Regezi J A, Sciubba J J, Pogrel M A. Atlas of Oral Pathology 2000. W B Saunders: Phila., p98-99

9. Assael L. Surgical management of odontogenic cysts and tumors. In Peterson L J et al:: Principles of Oral and Maxillofacial Surgery. vol 3, Lippincott, 1992, pp 694-697. 